\title{
Preparation of a plasma membrane-rich fraction from rat spermatozoa
}

\author{
R. Jones \\ AFRC Institute of Animal Physiology, Animal Research Station, 307 Huntingdon Road, \\ Cambridge CB3 OJQ, U.K.
}

\begin{abstract}
Summary. Monoclonal antibodies to antigens located on surface and intracellular membranes of spermatozoa from the rat cauda epididymidis have been used as probes to assess the purity of putative plasma membrane fractions. Spermatozoa were demembranated by shearing forces generated on a vortex-mixer. Immunofluorescence and ultrastructural analysis of vortex-mixed spermatozoa showed that they were denuded of approximately $90 \%$ of surface membrane. Areas of acrosomal membranes were also removed. Crude plasma membranes were recovered in low-speed wash fluids and fractionated on a $13-23 \%$ Nycodenz density gradient. Three bands containing membrane vesicles were resolved. Absorption curves and direct binding assays using monoclonal antibodies specific for acrosomal membranes, mitochondrial membranes and fibrous sheath showed relatively strong binding to bands 1 and 2 but weak binding to band 3. Conversely a monoclonal antibody specific for a surface membrane antigen bound strongly to band 3 and weakly to bands 1 and 2 . Identification on immunoblots of the antigens recognized by the monoclonal antibodies revealed that band 3 was positive for surface membrane antigens but gave no reaction for intracellular antigens. However, bands 1 and 2 were strongly positive for intracellular components. The results suggest that vortex-mixing is a simple and efficient means of removing the plasma membrane from spermatozoa and that a membrane fraction can be recovered from a Nycodenz density gradient that is enriched 40 - to 50 -fold in surface antigens.
\end{abstract}

\section{Introduction}

The surface membrane of mammalian spermatozoa has been investigated by a variety of ultrastructural, cytochemical and immunological procedures (e.g. Fawcett, 1975; Koehler, 1981; Friend, 1982). The evidence that has accrued from these investigations indicates that, whilst the general organization of proteins and lipids is similar to that in other eukaryotic cells, the sperm membrane possesses a number of unusual features. Some molecules are partitioned into separate domains overlying the head, midpiece and principal piece (Feuchter, Vernon \& Eddy, 1981; Myles, Primakoff $\&$ Bellve, 1981) whereas others are uniformly distributed and are free to move within a fluid environment (Gaunt, Brown \& Jones, 1983). Whilst these studies have shed some light on specific aspects of membrane structure they have provided relatively little information on its overall composition. To a large extent this is a reflection of the difficulty of purifying plasma membranes from spermatozoa for a detailed biochemical analysis and as a result our knowledge of the molecular structure of the sperm membrane lags considerably behind that on the erythrocyte or lymphocyte membrane.

Membrane preparations can be recovered from spermatozoa that have been disrupted by sonication, hypotonic shock, homogenization or nitrogen cavitation (Lunstra, Clegg \& Moore, 1974; Zahler \& Doak, 1975; Esbenshade \& Clegg, 1976; Gillis, Peterson, Russell, Hook \& Freund, 1978; Bradley \& Forrester, 1980; Peterson, Russell, Bundman \& Freund, 1980; Ivanov \& Profirov, 1981; Noland, Olson \& Garbers, 1983). Although all of these procedures release plasma membranes, 
membranes from intracellular organelles (e.g. acrosome, mitochondria) will also be present in varied amounts. The problem, therefore, is two-fold; first to develop a method for removing the surface membrane from spermatozoa without damaging intracellular organelles, and second, to assess the purity of any membrane fraction obtained in an objective and quantitative manner. The classical enzyme markers used for plasma membrane and mitochondria in other fractionation systems do not give satisfactory results when applied to spermatozoa (Noland et al., 1983; R. Jones, unpublished observations) and frequently preparations have been designated as plasma membrane by subjective and ill-defined criteria. We have developed a spectrum of monoclonal antibodies to surface and intracellular antigens on rat spermatozoa (Gaunt et al., 1983; Jones, Brown, Cran \& Gaunt, 1983) and in this work these monoclonal antibodies have been used as probes to assess the efficiency of different methods for removing the surface membrane from spermatozoa and for evaluating the purity of various membrane fractions by absorption assays and immunoblotting techniques.

\section{Materials and Methods}

Collection and washing of spermatozoa. Spermatozoa were flushed out of the cauda epididymidis of adult male rats (Wistar strain) by retrograde injection through the vas deferens of phosphatebuffered saline $\mathrm{pH} 7.4$ containing the proteinase inhibitors phenylmethyl-sulphonylfluoride (PMSF, $2 \mathrm{mM}$ ) and iodoacetamide (PBSI, $10 \mathrm{~mm}$ ). Spermatozoa were washed twice by centrifugation at $300 \mathrm{~g}$ for $7 \mathrm{~min}$ in round-bottomed test-tubes and gentle resuspension in PBSI to $2-5 \times 10^{7}$ cells $/ \mathrm{ml}$ (as determined by haemocytometer counts).

Production of monoclonal antibodies. Monoclonal antibodies were produced by immunizing female BALB/c mice with washed spermatozoa followed by fusion of lymphocytes with an NS-1 myeloma cell line as outlined by Gaunt et al. (1983). Using methods described previously (Jones et al., 1983) several monoclonal antibodies were selected as markers for membranes from various cell organelles. Briefly, intact or permeabilized (freeze-thawed twice) spermatozoa were stained with the monoclonal antibody followed by fluorescein-conjugated rabbit anti-mouse IgG (see below). If considerably $<90 \%$ of intact spermatozoa were positive but the proportion increased to $>90 \%$ with permeabilized cells then it was considered that the antigen was internal. This was subsequently verified at the ultrastructural level (Jones et al., 1983). The monoclonal antibodies used in this investigation were designated 2B1 (for surface membrane), 3D5 (for mitochondrial membrane), 5A5 (for fibrous sheath), 5B1 (for acrosomal membrane) and $1 \mathrm{~B} 6$ (for post-acrosomal surface membrane specifically). Data on 2B1, 3D5, SA5 and 1B6 have been published (Gaunt et al., 1983; Jones et al., 1983). The distribution of the antigen recognized by $5 \mathrm{~B} 1$ monoclonal antibody is shown in Fig. 1 ( $\mathrm{g} \& \mathrm{~h})$.

Immunofluorescence and electron microscopy. Antigens on spermatozoa were visualized by indirect immunofluorescence microscopy using monoclonal antibody (in culture supernatant) as first layer and fluorescein-conjugated rabbit anti-mouse IgG (FITC-RAM; Miles Scientific, PO Box 37, Stoke Poges, U.K.) as second layer reagent (Gaunt et al., 1983). Cells and membrane pellets were fixed for electron microscopy in $2 \%$ glutaraldehyde in $0.15 \mathrm{M}$-sodium chloride buffered with $0 \cdot 1 \mathrm{M}$-sodium cacodylate $\mathrm{pH} 7.4$ and processed as described previously (Jones et al., 1983).

Preparation of membrane fractions. Spermatozoa (in $\sim 3 \mathrm{ml}$ ) were transferred to a roundbottomed glass test-tube (height $110 \mathrm{~mm}$; i.d. $14 \mathrm{~mm}$ ) and demembranated by shearing forces generated on a standard laboratory vortex-mixer set at approximately 500 r.p.m. (Fisons Scientific, Loughborough, U.K.) for $2 \mathrm{~min}$ (see 'Results'). The spermatozoa were then washed twice by centrifugation at $300 \mathrm{~g}$ for $7 \mathrm{~min}$, and the supernatants were combined and cleared of residual spermatozoa and cytoplasmic droplets by filtration through an Amicon Microporous filter $(0.8 \mu \mathrm{m}$ pore size; $25 \mathrm{~mm}$ diam.; Amicon Corp., Danvers, MA, U.S.A.) fitted in a plastic Swinnex adaptor 
(Millipore, Cat. no. SX00 02500 ). The filtrate was adjusted to $10 \mathrm{ml}$ with PBSI and layered over $20 \mathrm{ml}$ of a linear gradient (13.8\% to $23 \%$ ) of isosmotic Nycodenz (Nyegaard \& Co. AS, PO Box 4220 , Oslo 4 , Norway) prepared by diluting a stock solution of $27.6 \%$ Nycodenz in 10 mM-Hepes $\mathrm{pH} 7 \cdot 4,3 \mathrm{~mm}-\mathrm{KCl}, 1 \mathrm{~mm}$-EDTA with the appropriate volume of $7 \cdot 5 \%$ sucrose in $10 \mathrm{mM}$-Hepes pH 7.4, 3 mM-KCl, 1 mM-EDTA. The gradient was prepared in a transparent cellulose nitrate tube (height $75 \mathrm{~mm}$; i.d. $25 \mathrm{~mm}$; capacity $35 \mathrm{ml}$ ) and centrifuged at 25000 r.p.m. (63000 g average) in a Beckman SW 25 rotor for $16 \mathrm{~h}$ at $10^{\circ} \mathrm{C}$. Opalescent bands containing membranes were aspirated with a Pasteur pipette, diluted with $10 \mathrm{~mm}$-Hepes $\mathrm{pH} 7.4$ and centrifuged at $100000 \mathrm{~g}$ for $60 \mathrm{~min}$ in a Beckman 50Ti rotor. Depending on the experiment, membrane pellets were then fixed in glutaraldehyde for electron microscopy, or resuspended in PBSI for absorption assays or solubilized directly in $62.5 \mathrm{~mm}$-Tris $-\mathrm{HCl} \mathrm{pH} \mathrm{7 \cdot 4/2 \%}$.SDS/4\% 2-mercaptoethanol for electrophoresis.

Electrophoresis. Proteins were separated by electrophoresis on reducing SDS polyacrylamide slab gels $(14 \mathrm{~cm} \times 9 \mathrm{~cm} \times 0.1 \mathrm{~cm})$ using a gradient of $8-16 \%$ polyacrylamide (Laemmli, 1970). Proteins were stained with $0.5 \%$ Coomassie Blue in methanol:acetic acid:water (40:7:53 by vol.) and destained in the same solvent without dye. Molecular weight standards $\left(M_{\mathrm{r}} 14000-94000\right)$ were purchased from Pharmacia Ltd (Uppsala, Sweden).

Extraction of spermatozoa with detergents. Washed spermatozoa were resuspended in $1 \mathrm{ml}$ of the following detergents made up to $0.5 \%$ in PBSI pH 7.4. Triton X-100 (Sigma Ltd), Nonidet P-40 (NP-40; Sigma Ltd), 3-[(-cholamidopropyl)-dimethylammonio]-1-propanesulphonate (CHAPS; Pierce \& Warriner, Chester, U.K.), decanoyl- $N$-methylglucamide (MEGA-10; Hildreth, 1982) and sodium deoxycholate $\mathrm{pH} 8.4$ (DOC; BDH Ltd, Poole, Dorset, U.K.). The sperm suspensions were placed on ice for $30 \mathrm{~min}$ and centrifuged at $10000 \mathrm{~g}$ for $15 \mathrm{~min}$, and solubilized proteins in the supernatant were removed for electrophoresis on reducing SDS polyacrylamide gels.

Binding of monoclonal antibodies to membrane fractions. Pellets of crude membranes or membrane fractions from density gradients were resuspended in $300 \mu 1$ PBSI and protein concentrations adjusted to $1 \mathrm{mg} / \mathrm{ml}$. An amount containing $300 \mu \mathrm{g}$ protein was made $0 \cdot 1 \%$ with bovine serum albumin (BSA, fraction V; Sigma), the volume adjusted to $500 \mu$ l and then divided into $5 \times 100 \mu \mathrm{l}$ aliquants. To each aliquant was added $200 \mu \mathrm{l}$ of HAT medium or culture supernatant containing monoclonal antibodies $2 \mathrm{~B} 1,3 \mathrm{D} 5,5 \mathrm{~A} 5$ or $5 \mathrm{~B} 1$, and the mixture was incubated at $4^{\circ} \mathrm{C}$ for $30 \mathrm{~min}$. Preliminary experiments showed that the concentration of antibody used was saturating. The suspensions were diluted with $10 \mathrm{ml}$ PBS $/ 0 \cdot 1 \% \mathrm{BSA}$, centrifuged at $100000 \mathrm{~g}$ for $60 \mathrm{~min}$ and the supernatants discarded. The walls of the centrifuge tube were wiped dry and the pellets resuspended in $200 \mu \mathrm{l}$ PBS $/ 0 \cdot 1 \%$ BSA containing $0.25 \mu \mathrm{Ci}^{125} \mathrm{I}$-labelled sheep anti-mouse $\mathrm{F}\left(\mathrm{ab}^{\prime}\right) 2$ ([ $\left.{ }^{125} \mathrm{I}\right]$ SAM; Amersham International, Bucks, U.K.). Suspensions were incubated at $4^{\circ} \mathrm{C}$ for $30 \mathrm{~min}$, washed with $10 \mathrm{ml} \mathrm{PBS} / 0 \cdot 1 \% \mathrm{BSA}$ as described above and bound radioactivity in the membrane pellets counted in a Nuclear Enterprises NE 1600 gamma counter. Protein concentration was estimated by a modified Lowry procedure (Hartree, 1972) or the method of Bradford (1976) using bovine serum albumin as standard.

Immunoblotting. Proteins separated on SDS polyacrylamide gels were blotted onto nitrocellulose $(0.45 \mu \mathrm{m}$; Anderman \& Co. Ltd, 145 London Road, Kingston-upon-Thames, U.K.) as described by Towbin, Staehelin \& Gordon (1979) for $18 \mathrm{~h}$ at $10 \mathrm{~V} / \mathrm{cm}$. Blots were blocked with $3 \%$ BSA in PBS for $1 \mathrm{~h}$ at $20^{\circ} \mathrm{C}$ and the antigen was detected by incubating the paper with $1: 3$ diluted culture supernatant for $1 \mathrm{~h}$ followed by 1:1000 diluted peroxidase-conjugated rabbit anti-mouse antibody (Miles). 4-Chloronaphthol (Sigma Ltd) was used as the final chromagen: $20 \mathrm{mg}$ reagent were dissolved in $10 \mathrm{ml}$ methanol and added to $50 \mathrm{ml}$ PBS followed by $30 \mu \mathrm{H}_{2} \mathrm{O}_{2}(30 \%)(\mathrm{w} / \mathrm{v})$. The reaction was stopped by washing in distilled water and drying the paper.

Absorption assays. Monoclonal antibody supernatants were diluted $1 / 8$ with $\mathrm{PBSI} / 0 \cdot 1 \%$ BSA and $200 \mu \mathrm{l}$ aliquants were mixed with $200 \mu \mathrm{l}$ of sperm suspensions (intact or demembranated by 
vortex mixing) or membranes containing 1, 5, 25, 125, 625 or $3125 \mu$ g protein (Hartree, 1972). All dilutions were made in PBSI with BSA added to $0 \cdot 1 \%$. Preliminary experiments showed that any decrease in antibody titre was linear below a $1 / 8$ dilution of culture supernatant. Mixtures were incubated for $45 \mathrm{~min}$ at $4^{\circ} \mathrm{C}$, centrifuged at $10000 \mathrm{~g}$ for $10 \mathrm{~min}$ and the amount of antibody remaining in supernatants was measured using intact or demembranated spermatozoa as targets with $\left[{ }^{125} 1\right]$ SAM as second layer antibody as described by Jones et al. (1983).

\section{Results}

\section{Demembranation of spermatozoa}

Experiments using immunofluorescence. Initially several procedures were examined for their efficiency to demembranate spermatozoa. Sonication, freeze-thawing and hypotonic shock all disrupted spermatozoa to varied extents and released surface membrane as shown by the decrease in their ability to stain with 2B1 monoclonal antibody (marker for surface membrane). However, morphological examination with the light and electron microscope showed that these procedures caused significant damage to intracellular organelles, e.g. rupture and detachment of mitochondria and fragmentation of the flagellum (results not shown). Since it is desirable that as little intracellular material as possible should be released, other methods were investigated. It was found that vortex-mixing generated shear forces that were sufficient to remove surface membrane from spermatozoa but were insufficient to fracture the cell. This was demonstrated by staining spermatozoa with $2 \mathrm{Bl}$ monoclonal antibody/FITC-RAM and then vortex-mixing. Before vortex-mixing the antigen showed a characteristic patchy distribution all over the plasma membrane of spermatozoa (Fig. 1a; see also Gaunt et al., 1983). The binding of 2B1 to the sperm head is too weak to produce a photographic image but $2 \mathrm{~B} 1$ antigen is not excluded from the head domain. A time-course of vortex-mixing showed that, after $10 \mathrm{sec}, 90 \pm 1 \cdot 2 \%$ (mean \pm s.d.; 3 experiments) of cytoplasmic droplets had been removed (these droplets remained intact as viewed by phase-contrast microscopy and quantitative numbers could be recovered on sucrose gradients (Garbers, Wakabayashi \& Reed, 1970 ) ), after $30 \sec 81 \% \pm 2 \cdot 3$ of spermatozoa were fluorescent only over the midpiece region (Fig. lb) and after 2 min all spermatozoa were negative (Figs 1c \& d). Instead, large numbers of small fluorescent vesicles were present that were dispersed randomly throughout the field of view (Fig. 1c). If the latter spermatozoa were stained subsequently with $2 \mathrm{~B} 1$ monoclonal antibody/FITCRAM no fluorescence was observed, suggesting that surface membrane had been removed and that the effect was not caused simply by antibodies falling off the membrane. The crude membrane pellet recovered (see later) from spermatozoa vortex-mixed for 2 min contained $80-90 \%$ of the total membrane-bound protein that it was possible to release by this procedure (results not shown).

As mentioned above, $2 \mathrm{~B} 1$ monoclonal antibody does not bind strongly to the sperm head and to assess further the removal of plasma membrane overlying this region spermatozoa were stained

Fig. 1. (a)-(c) Distribution of immunofluorescence on the surface membrane of spermatozoa stained with 2B1 monoclonal antibody/FITC-RAM and then vortex-mixed for (a) 0 min; (b) $0.5 \mathrm{~min}$; (c) 2 min. (d) Phase-contrast view of (c). Similar numbers of spermatozoa were present in each field of view. Note that in (b) only the outline of the midpiece is discernible whilst in (c) no fluorescence is associated with spermatozoa. Instead, patches of fluorescence are distributed randomly throughout the field of view. (e)-(f) Immunofluorescence on spermatozoa stained with 1 B6 monoclonal antibody/FITC-RAM and vortex-mixed for $0 \min (e)$, or $2 \min (f)$. The antigen is restricted to the surface membrane over the post-acrosomal domain. (g) Immunofluorescence on spermatozoa stained with 5B1 monoclonal antibody (FITC-RAM). The antigen is restricted to the acrosome. About $28 \%$ of freshly washed spermatozoa stain with 5B1/FITC-RAM but this increases to $100 \%$ if spermatozoa are permeabilized by freezethawing as shown. The antigen is present on the inner and outer acrosomal membranes. Bar $=5 \mu \mathrm{m}$. (h) Phase contrast view of $(\mathrm{g})$. 

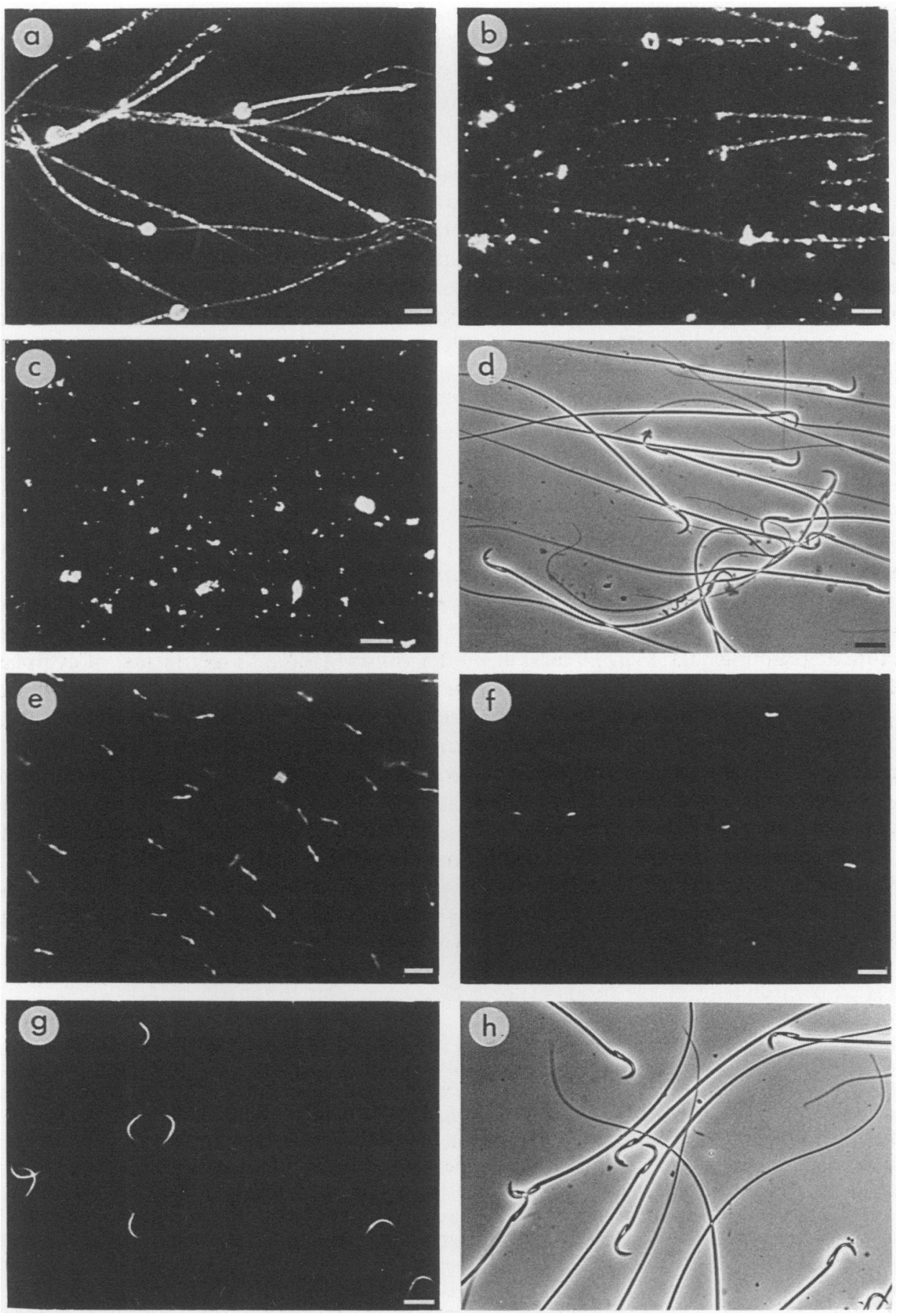
first with a monoclonal antibody (designated 1B6) that recognizes an antigen localized in the postacrosomal domain (Gaunt et al., 1983). Before vortex-mixing all spermatozoa showed positive fluorescence but after vortex-mixing $76 \pm 3 \cdot 1 \%$ (mean \pm s.d. of 5 experiments) of spermatozoa were no longer fluorescent and the remaining spermatozoa showed considerably reduced fluorescence over the post-acrosomal region (Figs le-f) ). Therefore, although vortex-mixing for $2 \mathrm{~min}$ does not dislodge all the surface membrane overlying the post-acrosomal region from all spermatozoa, it nonetheless removes it from a substantial proportion of cells.

Ultrastructural observations. Electron microscopy of spermatozoa before vortex-mixing showed the presence of plasma membrane on all cells examined. Typically, it had a ruffled appearance over the flagellum (Fig. 2a) but on the head it was more closely apposed to acrosomal and nuclear membranes. However, after vortex-mixing for $0.5 \mathrm{~min}$ most spermatozoa had lost substantial areas of their surface membrane (Fig. 2b) and after 2 min all spermatozoa were devoid of plasma membrane overlying the flagellum (Fig. 2c). There was no evidence for damage to intracellular organelles such as mitochondria, fibrous sheath, outer dense fibres or axonemal filaments (Fig. 2c, insert). In the head region, however, it was evident that a variable amount of acrosomal membranes had been removed in addition to the plasma membrane (Fig. $2 \mathrm{~d}-\mathrm{f}$ ). This was particularly evident over the rostral region of the acrosome where gaps or holes were present in the outer acrosomal membrane (Fig. 2f).

\section{Recovery and fractionation of membranes}

Membrane vesicles released in this fashion were recovered by 3 low speed washes $(300 \mathrm{~g}$ for $7 \mathrm{~min}$ ) of vortex-mixed spermatozoa. The efficiency of recovery was estimated by labelling spermatozoa with $2 \mathrm{Bl}$ monoclonal antibody/[125I]SAM followed by demembranation as described above. In a typical experiment $77 \%$ of the total membrane-bound label was recovered in the first washfluid, $13 \%$ in the second wash-fluid and $2 \%$ in the third wash-fluid. Therefore, 2 washes will recover $>85 \%$ of surface membrane vesicles. Residual spermatozoa present in the pooled wash fluids were then removed by filtration through an Amicon microporous filter $(0.8 \mu \mathrm{m}$ pore size). This filtration step also removed cytoplasmic droplets as these organelles were not observed in the filtrate or in electron micrographs of crude membranes (see later). Subsequent examination of washes of the filter by phase-contrast microscopy revealed the presence of large numbers of intact droplets as well as residual spermatozoa (results not shown). Filtration is therefore a useful clean-up step before fractionation on a density gradient. It is possible that filtration also removes large sheets or clumps of membranes but this is more likely to influence yield than have any selective effect. The recovery

Fig. 2. Electron micrographs of spermatozoa before and after vortex-mixing. (a) Longitudinal section through the midpiece of a spermatozoon before vortex-mixing. Note the presence of an intact plasma membrane $(\mathrm{PM}), \times 32000$. (b) Midpiece region of spermatozoa vortex-mixed for $0.5 \mathrm{~min}$ (equivalent to those of spermatozoa shown in Fig. 1b). The upper spermatozoon has an intact plasma membrane whereas the lower spermatozoon has lost most of its plasma membrane. $\times 18000$. (c) Typical longitudinal section through the midpiece of a spermatozoon vortex-mixed for 2 min (equivalent to spermatozoa shown in Figs 1c \& 1d). The plasma membrane is absent but the mitochondria appear undamaged. Insert shows transverse section to illustrate outer dense fibres and axonemal filaments. $\times 28000$. (d) Section through the equatorial segment of the acrosome of a spermatozoon vortex-mixed for $2 \mathrm{~min}$. The plasma membrane has been removed but the outer acrosomal membrane (OAM), inner acrosomal membrane (IAM) and outer leaflet of the nuclear envelope (OLNE) are still present. $\times 80000$. (e) Transverse section through rostral region of a sperm head after vortex-mixing for 2 min. The PM, IAM and OAM have been removed from the left lateral edge but the IAM and OAM are still present along the right lateral edge. $\mathbf{N}=$ nucleus; $\mathbf{P}=$ perforatorial substance. $\times 32000$. (f) Rostral edge of spermatozoon vortex-mixed for $2 \mathrm{~min}$. The plasma membrane is absent as are pieces of the OAM. The IAM is intact over the perforatorial substance. $\times 60000$. 

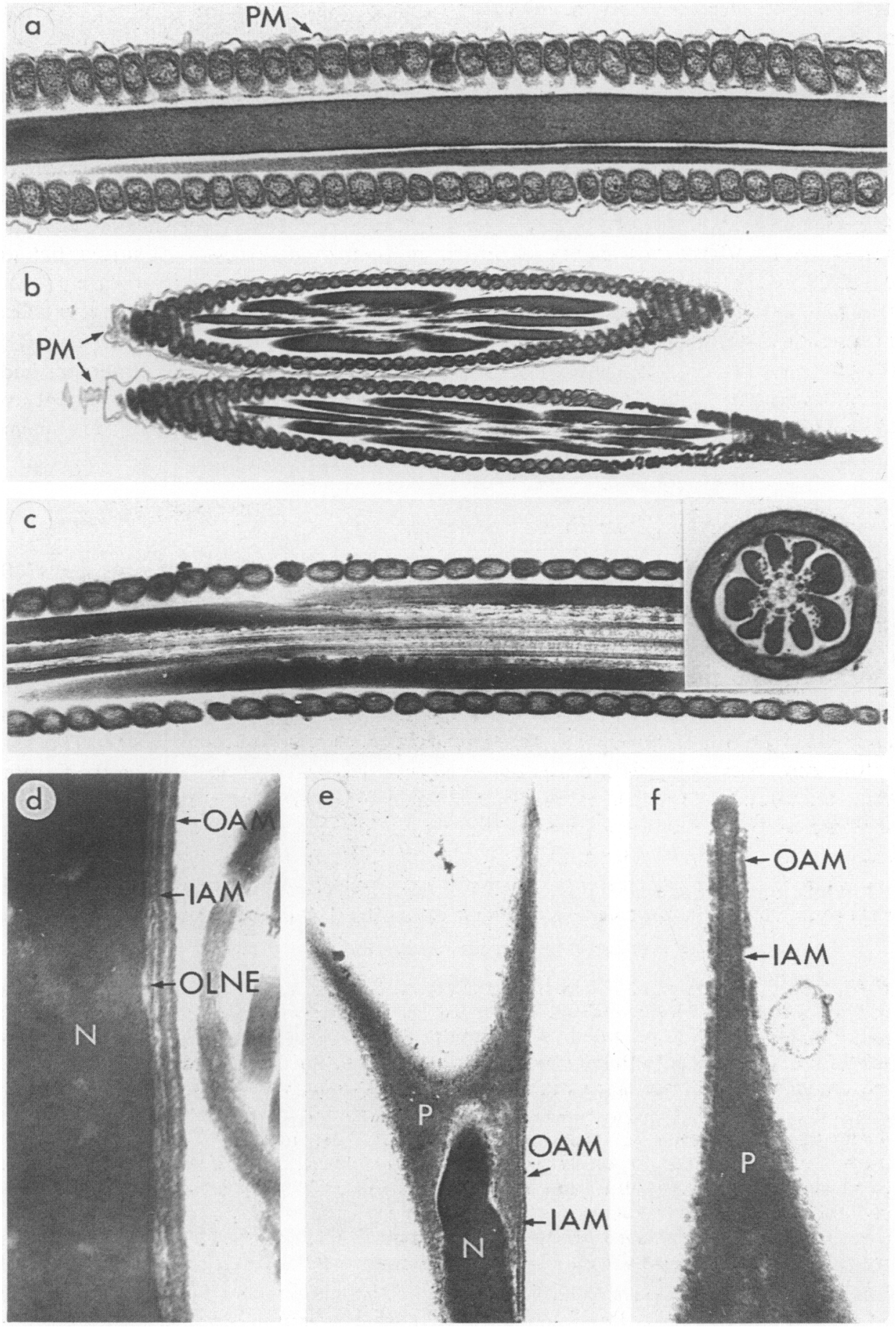

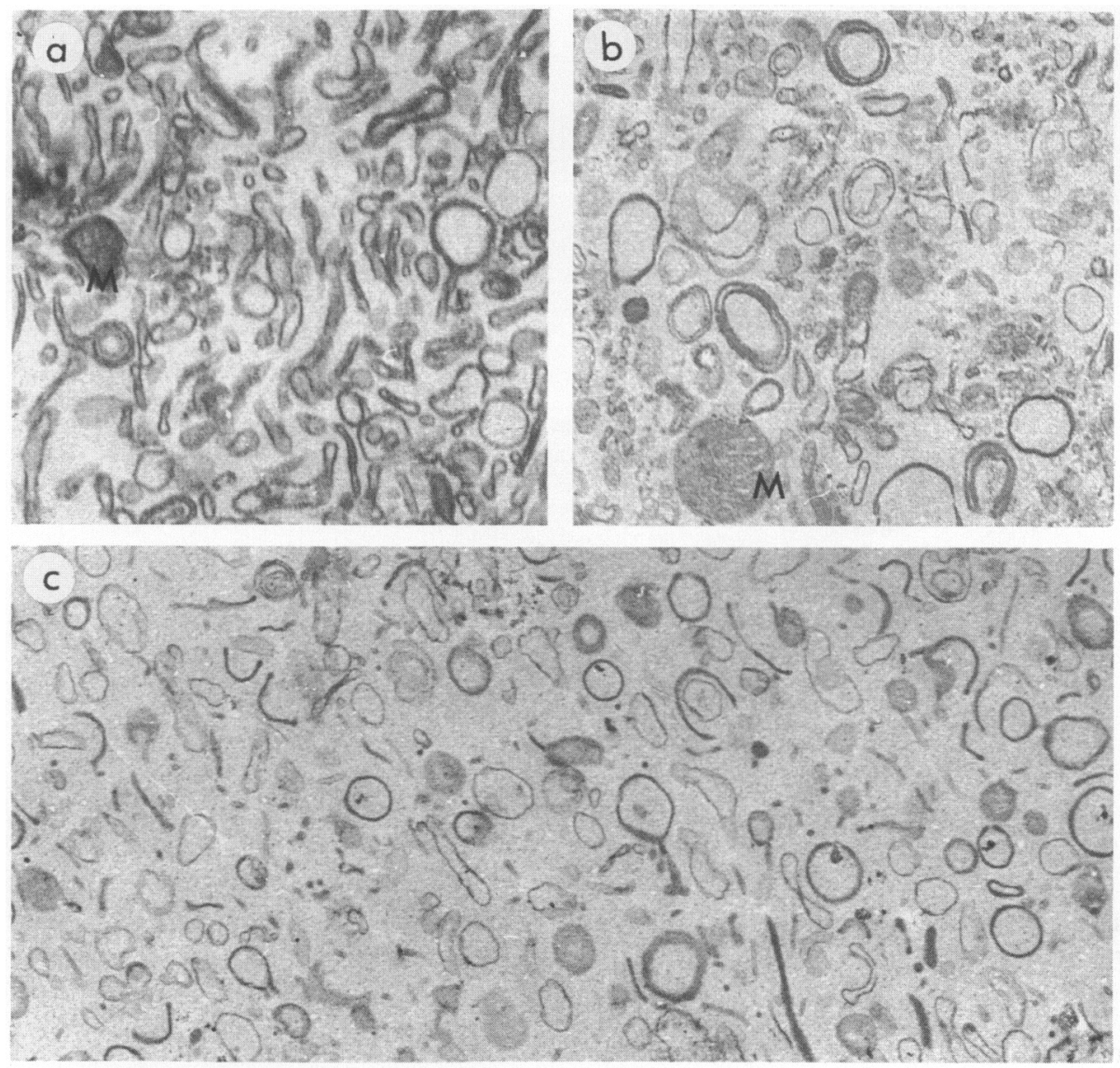

Fig. 3. Electron micrographs of (a) crude membranes, (b) membranes in $\mathbf{B} 1+\mathbf{B} 2$, and (c) membranes in $\mathbf{B} 3 . \mathbf{M}=$ mitochondrial remnant. $\times 28000$.

of 2B1 monoclonal antibody $\left./ \mathrm{I}^{125} \mathrm{I}\right] \mathrm{SAM}$-labelled membranes in the filtrate was $\sim 77 \%$, representing $73 \%$ of the original surface membrane. The preparation of membranes obtained after passage through the Amicon filter is referred to as crude membranes.

Centrifugation of the suspension of crude membranes through a $13.8-23 \%$ gradient of isomotic Nycodenz produced 3 bands containing membrane vesicles. Band 1 (B1) formed at the interface between PBSI and $13.8 \%$ Nycodenz and contained $10-15 \%$ of total membrane protein applied. Since it was difficult to aspirate separately it was pooled with the broader band 2 (B2) which formed between 14 and $15.5 \%$ of Nycodenz. Band 3 (B3) was located near the bottom of the gradient and contained between 40 and $50 \%$ of the total membrane protein applied.

\section{Electron microscopy of membrane fractions}

Ultrastructural examination of the crude membrane pellet revealed a heterogeneous population of empty membrane-bound vesicles, collapsed vesicles and vesicles within vesicles (Fig. 3a). Cytoplasmic droplets were never observed. Membrane vesicles in B1 + B2 were, if anything, more heterogeneous and fragments of mitochondria were present very occasionally (Fig. 3b). B3 membrane vesicles on the other hand appeared more homogeneous although of varied size and shape (Fig. 3c). However, on the basis of this evidence alone it was not possible to assess the relative 

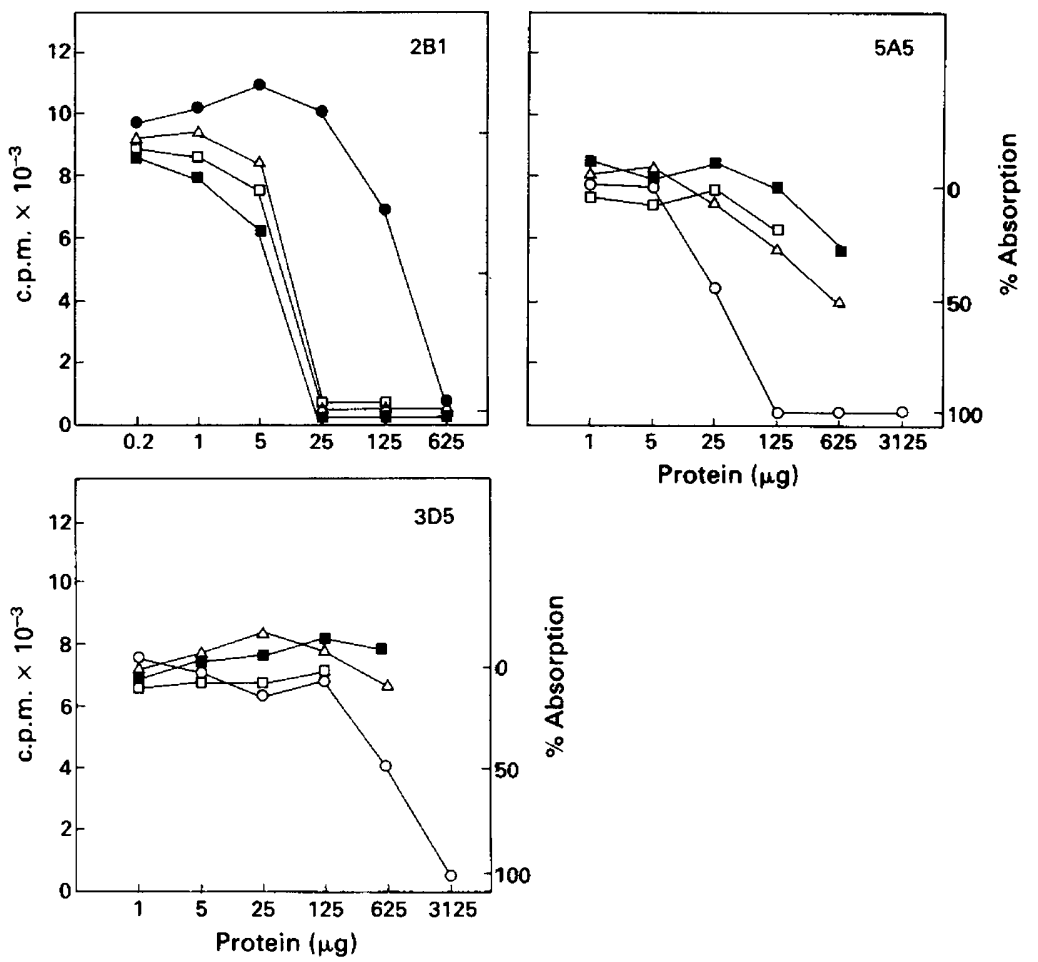

Fig. 4. Relative amounts of $2 \mathrm{~B} 1,5 \mathrm{~A} 5$ or $3 \mathrm{D} 5$ antigens on intact spermatozoa $(\bullet)$, demembranated spermatozoa $(O)$, crude membranes $(\triangle)$, B1 + B2 membranes $(\square)$ and B3 membranes $(\square)$ as measured by absorption assays. Constant amounts of antibody were absorbed with serial 5-fold dilutions of spermatozoa or membranes and remaining antibody assayed by an indirect radioactive binding assay as described in 'Material and Methods'. Percentage absorption values give the results of binding assays without prior absorption $(0 \%)$ or using no monoclonal antibody $(100 \%)$.

enrichment of the various fractions in surface membrane components. This was done using monoclonal antibodies as described below.

\section{Assessment of purity of membrane fractions}

The degree of enrichment of membrane fractions in surface components was assessed by measuring the binding of organelle-specific monoclonal antibodies to spermatozoa and membranes using 3 different procedures (absorption assays, direct binding assays and immunoblotting).

Absorption assays. Complete absorption by intact spermatozoa of $2 \mathrm{~B} 1$ monoclonal antibody from a $1 / 8$ diluted culture supernatant was reached with $625 \mu \mathrm{g}$ total protein (Fig. 4). By contrast, as little as $25 \mu \mathrm{g}$ of crude membrane protein was required to achieve $100 \%$ absorption, indicating an approximate 25 -fold enrichment of surface membrane relative to whole cells. Membranes in $\mathrm{B} 1+\mathrm{B} 2$ and B3 were not significantly different from crude membranes in this assay system although there was always a tendency for B3 membranes to absorb more strongly.

The converse absorption pattern was obtained with 5A5 and 3D5 monoclonal antibodies (Fig. 4). With 5A5 monoclonal antibody, demembranated spermatozoa absorbed strongly to reach $100 \%$ levels with $125 \mu \mathrm{g}$ protein but only $50 \%$ and $70 \%$ absorption could be achieved with 5 times 


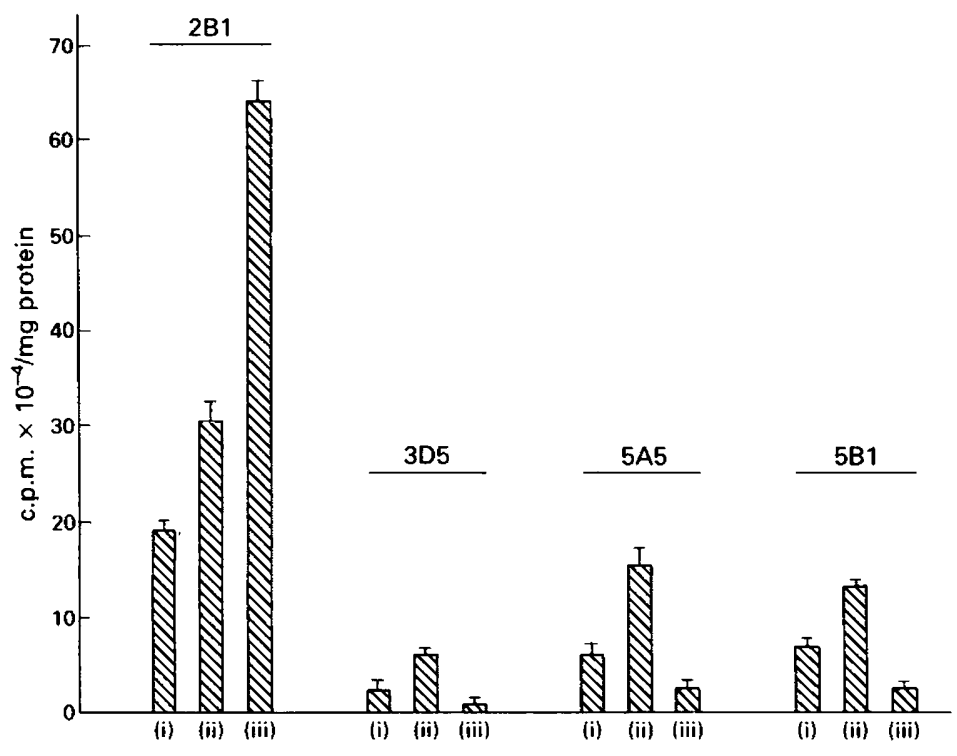

Fig. 5. Binding of monoclonal antibodies 2B1 (surface membrane), 3D5 (mitochondrial membrane), 5A5 (fibrous sheath) and 5B1 (acrosomal membrane) to crude membranes (i), membranes in B1 + B2 (ii) and membranes in B3 (iii). Membranes were incubated with monoclonal antibody culture supernatants followed by $\left[{ }^{125} \mathrm{I}\right] \mathrm{SAM}$ as described in 'Materials and Methods'. Values shown are the means \pm s.d. of 3 separate membrane preparations.

$(625 \mu \mathrm{g})$ the amount of crude membranes and B3 membranes respectively. It was not possible to obtain significant absorption values for $\mathrm{B} 1+\mathrm{B} 2$ membranes because of the difficulties of collecting more than $125 \mu \mathrm{g}$ material but the available data indicated that they were comparable to crude membranes. The 3D5 monoclonal antibody showed a similar pattern to 5A5 except that larger $(3125 \mu \mathrm{g})$ amounts of demembranated spermatozoa were required for complete absorption (Fig. 4). This may be due to a lower number of binding sites and/or affinity of the antibody. Because of this problem it was not possible to obtain $100 \%$ absorption values for membrane fractions. Nevertheless, it was clear that $50 \%$ absorption was achieved with $625 \mu \mathrm{g}$ spermatozoa whereas an equivalent amount of $\mathrm{B} 3$ or crude membranes remained at $0 \%$.

The 5B1 monoclonal antibody did not give satisfactory absorption curves. We attribute this to the small area of the spermatozoon (i.e. acrosome) that binds the antibody and hence prohibitively large amounts of material would be required to obtain significant absorption. Therefore, the degree of contamination by acrosomal membranes had to be assessed by direct binding assays and immunoblotting procedures.

Direct binding assays. Direct binding of 2B1 monoclonal antibody to B3 membranes was about 3 times greater than to crude membranes (Fig. 5). However, with 3D5, 5A5 and 5B1 monoclonal antibodies, binding to $\mathrm{B} 3$ membranes was 2- to 3-fold less than to crude membranes, although the $\mathrm{B} 1+\mathrm{B} 2$ membranes showed an increase in specific activity. These results suggest that B3 membranes are enriched about $2-3$-fold in surface membranes relative to crude material and depleted by a similar order of magnitude in intracellular antigens.

Immunoblotting. The distribution of antigens recognized by $2 \mathrm{~B} 1,5 \mathrm{~A} 5$ and $5 \mathrm{~B} 1$ monoclonal antibodies on immunoblots of membrane proteins separated on SDS polyacrylamide gels is shown in Fig. 6. The 3D5 monoclonal antibody did not give a reaction on blots. The $2 \mathrm{~B} 1$ monoclonal antibody recognized a protein with an $M_{\mathrm{r}}$ of $\sim 40000$ in crude membrane preparations. This antigen 

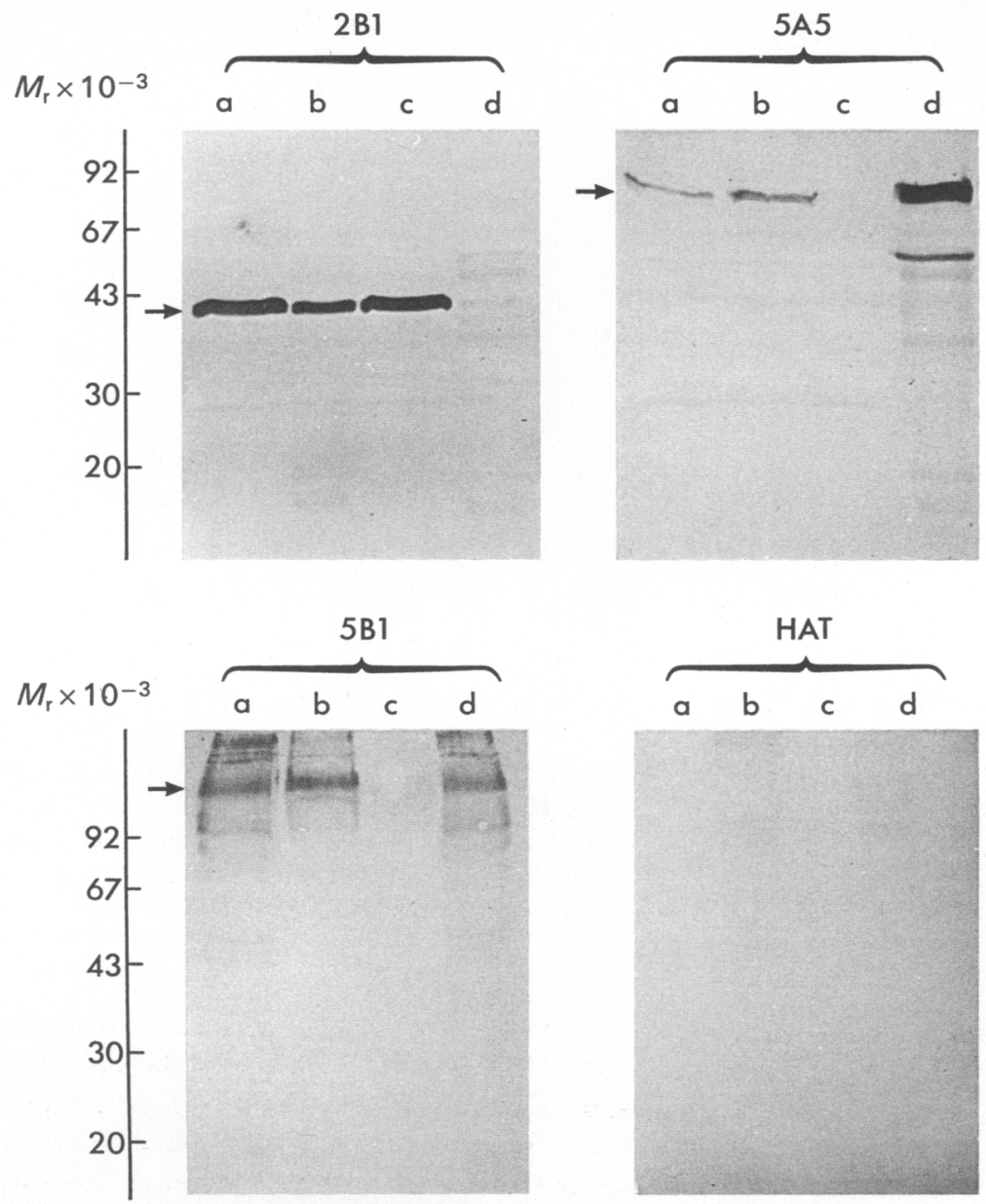

Fig. 6. Distribution of antigens recognized specifically by monoclonal antibodies $2 B 1,5 A 5$ and $5 \mathrm{~B} 1$ between (a) crude membranes, (b) membranes in B1 + B2, (c) membranes in B3, and (d) SDS extracts of demembranated spermatozoa. Antigens were detected on immunoblots as described in 'Materials and Methods'. Arrows indicate the major antigen recognized by each monoclonal antibody. About $30 \mu \mathrm{g}$ protein were applied to each track and values given are approximate $M_{\mathrm{r}}$ values. HAT $=$ culture supernatant minus antibody.

was also detected in B1 + B2 and B3 membranes but not in SDS extracts of demembranated spermatozoa. However, monoclonal antibody 5A5 showed a strong reaction over an antigen with an $M_{\mathrm{r}}$ of $\sim 85000$ in extracts of demembranated spermatozoa. A weak reaction was detected over this antigen in crude membranes and in B1 + B2 membranes but no reaction was observed in B3 membranes. Preliminary experiments in which various amounts of crude membrane protein on blots were probed with 5A5 monoclonal antibody showed that a 3-fold reduction in reaction intensity to that shown in Fig. 6, 5A5 track (a), was still detectable. The intensity of the reaction was noticeably stronger over demembranated spermatozoa, a distribution that is consistent with the view that the antibody is recognizing a protein in the fibrous sheath. Olson, Hamilton \& Fawcett (1976) have reported a $M_{\mathrm{r}}$ of 80000 for this protein.

Similarly, 5B1 monoclonal antibody recognized primarily a $160000 M_{\mathrm{r}}$ antigen in crude membranes, in B1 + B2 membranes and in SDS extracts of demembranated spermatozoa, but no reaction was detected in $\mathrm{B} 3$ membranes (Fig. 6). 


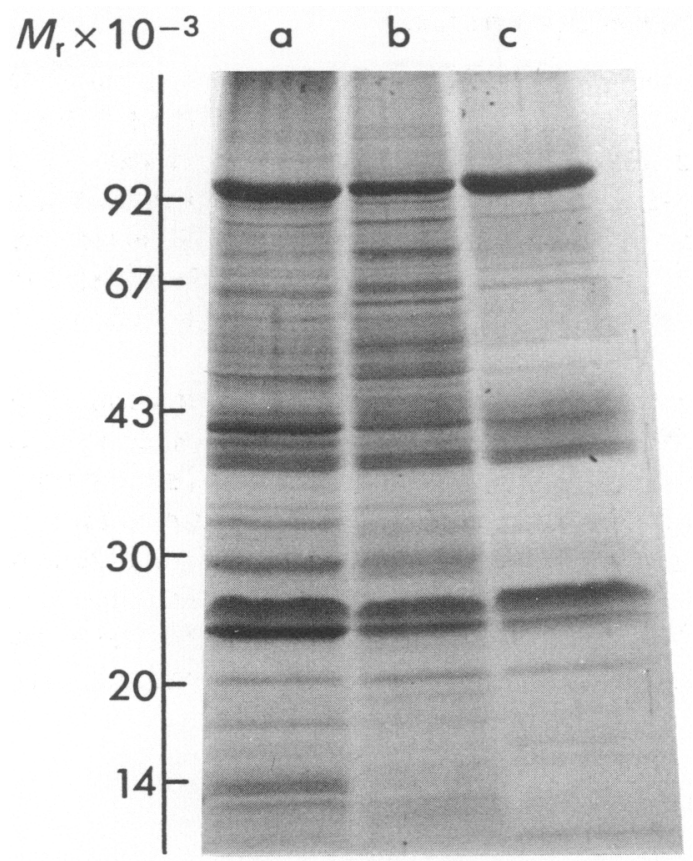

Fig. 7. Separation of proteins in (a), crude membranes, (b) membranes in B1 + B2, and (c) membranes in B3 on a reducing SDS polyacrylamide gel. Proteins were stained with Coomassie Blue and each track received $\sim 30 \mu \mathrm{g}$ protein. The $M_{\mathrm{r}}$ values of protein standards (Pharmacia Ltd) are shown.

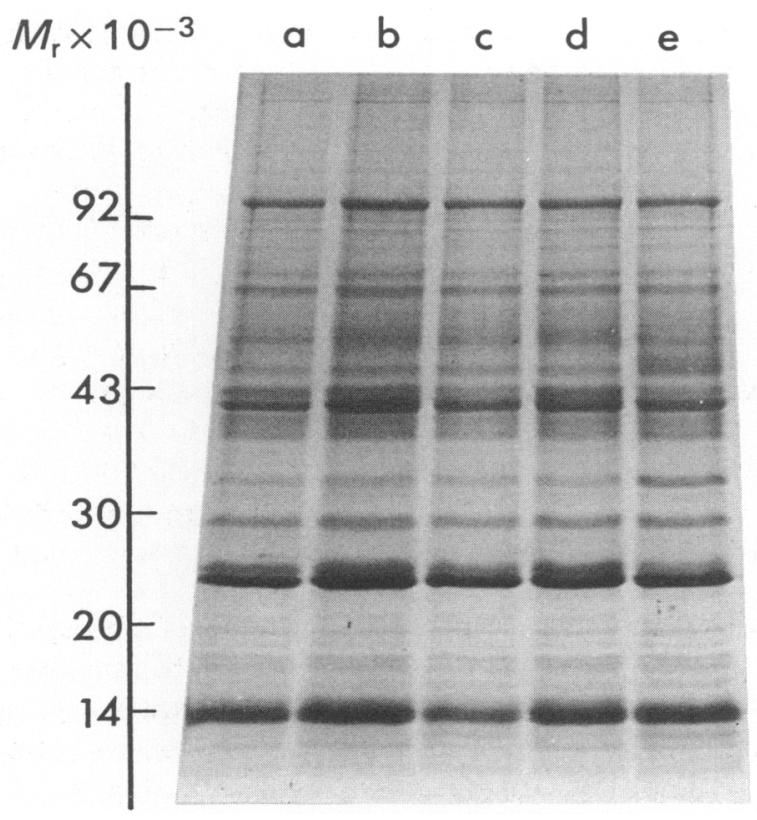

Fig. 8. SDS polyacrylamide gel electrophoresis of proteins solubilized directly from washed rat spermatozoa with (a) Triton X-100, (b) NP-40, (c) CHAPS, (d) MEGA-10, and (e) DOC. Proteins were stained with Coomassie Blue and each track received $\sim 30 \mu \mathrm{g}$ protein. The $M_{\mathrm{r}}$ values of standards are shown. 


\section{Electrophoresis of proteins in membrane fractions}

Electrophoresis of proteins in membrane fraction on reducing SDS polyacrylamide gels revealed a wide range of proteins with $M_{\mathrm{r}}$ values of 13000 to 150000 (Fig. 7a). The major proteins in this fraction, as assessed by uptake of Coomassie Blue stain, have $M_{\mathrm{r}}$ values of $23000-24000$, $40000-45000$ and $100000-120000$. In B1 + B2 the relative amounts of these proteins decreased noticeably with a concomitant increase in the proportion of several proteins with $M_{\mathrm{r}}$ ranging from 45000 to 85000 (Fig. 7b). However, B3 was significantly depleted in the latter proteins and the major components were again those with $M_{\mathrm{r}}$ values of $23000-24000,40000-45000$ and $100000-120000$ (Fig. 7c).

It was of interest to compare the profile of proteins in membrane fractions (obtained in this manner) with that found by extracting spermatozoa directly with detergents. It is to be expected that detergent extracts will contain, in addition to surface membrane proteins, water-soluble proteins and a variable amount of components from intracellular membranes. As shown in Fig. 8 the profile of proteins in detergent extracts is close to that found in a crude membrane fraction. The main point of variance is that several additional proteins are present (e.g. at $M_{\mathrm{r}} 14000$ ) which, for reasons given above, is not unexpected. It is also evident that there is little difference between Triton X-100, NP-40, CHAPS, MEGA-10 and DOC in the type and relative amounts of proteins solubilized.

\section{Discussion}

The evidence presented in this investigation suggests that vortex-mixing is a simple and efficient method for removing plasma membranes from rat spermatozoa and that centrifugation of the membrane preparation so produced through a density gradient of Nycodenz yields a fraction (designated B3) that is enriched in surface membrane components.

The unusual fragility of rat spermatozoa precludes methods such as sonication or mechanical homogenization as a means of preparing surface membranes. These procedures readily decapitate cells and fragment the flagellum, producing a very heterogeneous mixture of membrane vesicles and remnants of organelles such as mitochondria, fibrous sheath and outer dense fibres. Vortexmixing on the other hand generates relatively mild shearing forces that are sufficient to remove surface membranes and acrosomal membranes yet do not break up spermatozoa or cause excessive release of intracellular organelles. Therefore, vortex-mixing compares favourably with the nitrogen cavitation procedure which has been used for demembranating boar and bull spermatozoa (Peterson et al., 1980; Noland et al., 1983). Nitrogen cavitation has the advantage of releasing only small amounts of acrosomal membranes but has the disadvantage of removing selectively surface membranes over limited areas of the head and flagellum so that a fully representative preparation is not obtained. In addition, nitrogen cavitation breaks bull spermatozoa in the principal piece region, increasing the risk of detaching intracellular organelles (Noland et al., 1983). Controlled vortexmixing overcomes these problems to a large extent as it dislodges $\sim 90 \%$ of the plasma membrane as shown by staining with $2 \mathrm{~B} 1$ monoclonal antibody (surface marker) and does not fracture the flagellum.

This vortexing protocol also seems suitable for spermatozoa of other species; a crude membrane pellet can be obtained from boar and ram spermatozoa using the procedure described for the rat (R. Jones, unpublished observations). The method may have general application since even crude membrane preparations are enriched 25 -fold over starting material. This compares favourably with the 20 -fold enrichment reported by Gillis et al. (1978) and Peterson et al. (1980) after fractionation of boar sperm membranes on density gradients and the 15- to 25-fold enrichment commonly found for liver and epithelial cell plasma membrane preparations by Neville (1976).

A major problem in developing methods for preparing plasma membranes from spermatozoa has been that of measuring purity. In many cases the source of the membranes has been inferred 
from ultrastructural examination of disrupted spermatozoa rather than an objective appraisal of the membrane pellet itself. The weakness of this approach is illustrated in the present work by the absence of any ultrastructural evidence for damage to mitochondria or fibrous sheath following vortex-mixing, yet sensitive immunoblotting techniques detected significant amounts of these organelles in crude membrane preparations. In the present method the relative binding of organellespecific monoclonal antibodies to membrane fractions has allowed a direct assessment of purity and of the derivation of extraneous material. The notion that B3 membrane vesicles are enriched in plasma membranes is supported by: (1) specific activity of binding of monoclonal antibody markers for surface membrane is increased about 3-fold in B3 relative to crude membranes. Conversely, markers for intracellular antigens are reduced 2-4-fold; (2) immunoblots of proteins in membrane fractions show that intracellular antigens are not detectable in B3 but are present in B1 + B2; (3) electrophoretic analysis of proteins in each fraction reveals that B3 is enriched in certain classes of proteins that are less well represented in $\mathrm{B} 1+\mathrm{B} 2$ and vice versa. It is possible that other intracellular antigens, to which we do not have specific antibody markers, are present in B3 but it is reasonable to presume that their contribution to the overall protein pattern must be small since the amounts of the major potential contaminants (mitochondria, fibrous sheath and acrosomal membranes) have been reduced considerably.

The finding that rat sperm surface membranes have a higher density on Nycodenz gradients than do intracellular membranes contrasts with that reported for bull and boar sperm plasma membranes on sucrose gradients (Gillis et al., 1978; Peterson et al., 1980). This may be a genuine species difference or it may be a property of the Nycodenz gradient itself which gives cleaner and better separation of membrane vesicles than sucrose gradients ( $R$. Jones, unpublished observations).

A limitation of the present method, and of all methods for preparing plasma membranes from cells, is that loosely bound 'extrinsic' proteins may be lost during some stage of membrane isolation. Evaluating the magnitude of this problem will require careful investigation but the finding that the profile of proteins in isolated membrane fractions is not radically different from direct detergent extracts (in which, presumably, all surface components are retained) suggests that only minor constituents of the membrane are affected. Therefore, the protocol outlined above should provide a platform to characterize further biochemical changes to the surface membrane of rat spermatozoa undergoing developmental changes such as maturation and capacitation.

I thank Mr K. I. von Glos for technical assistance, Dr D. G. Cran for performing the electron microscopy and Dr S. J. Gaunt and Mr C. R. Brown for helpful discussions.

Note added in proof. Amincon Corp. have discontinued manufacture of the microporous filter $(0.8 \mu \mathrm{m}$ pore size) used in this investigation. A suitable alternative is the GA-4 filter (cat. no. 60109) produced by Gelman Sciences (10 Harrowden Road, Northampton NN4 0EZ, U.K.), although the recovery of membranes is reduced from $73 \%$ to $\sim 56 \%$.

\section{References}

Bradford, M.M. (1976) A rapid and sensitive method for the quantitation of microgram quantities of protein utilizing the principle of protein-dye binding. Analyt. Biochem. 72, 248-254.

Bradley, M.P. \& Forrester, I.J. (1980) A $\left[\mathrm{Ca}^{2+}+\right.$ $\left.\mathrm{Mg}^{2+}\right]$-ATP and active $\mathrm{Ca}^{2} \uparrow$ transport in the plasma membranes isolated from ram sperm flagella. Cell Calcium 1, 381-390.

Esbenshade, K.L. \& Clegg, E.D. (1976) Electrophoretic characterization of proteins in the plasma membrane of porcine spermatozoa. J. Reprod. Fert. 47, 333-337.
Fawcett, D.W. (1975) The mammalian spermatozoon. Devl Biol. 44, $394-436$.

Feuchter, F.A., Vernon, R.B. \& Eddy, E.M. (1981) Analysis of the sperm surface; topographically restricted antigens appearing in the epididymis. Biol. Reprod. 24, $1099-1110$.

Friend, D.S. (1982) Plasma-membrane diversity in a highly polarized cell. J. Cell Biol. 93, 243-249.

Garbers, D.L., Wakabayashi, T. \& Reed, P.W. (1970) Enzyme profile of the cytoplasmic droplet from bovine epididymal spermatozoa. Biol. Reprod. 3, $327-337$. 
Gaunt, S.J., Brown, C.R. \& Jones, R. (1983) Identification of mobile and fixed antigens on the plasma membrane of rat spermatozoa using monoclonal antibodies. Expl Cell Res. 144, 275-284.

Gillis, G., Peterson, R.N., Russell, L., Hook, L. \& Freund, M. (1978) Isolation and characterization of membrane vesicles from human and boar spermatozoa: methods using nitrogen cavitation and ionophore induced vesiculation. Prep. Biochem. 8, 363-378.

Hartree, E.F. (1972) Determination of protein: a modifcation of the Lowry method that gives a linear photometric response. Analyt. Biochem. 48, 422-427.

Ivanov, N. \& Profirov, Y. (1981) Isolation of plasma membranes from ram spermatozoa by a two-phase polymer system. J. Reprod. Fert. 63, 25-29.

Jones, R., Brown, C.R., Cran, D.G. \& Gaunt, S.J. (1983) Surface and internal antigens of rat spermatozoa distinguished using monoclonal antibodies. Gamete Res. 8, 255-265.

Koehler, J.K. (1981) Lectins as probes of the spermatozoon surface. Archs Androl. 6, 197-217.

Laemmli, U.K. (1970) Cleavage of structural proteins during the assembly of the head of bacteriophage T4. Nature, Lond. 227, 680-685.

lunstra, D.D., Clegg, E.D. \& Moore, D.J. (1974) Isolation of plasma membrane from porcine spermatozoa. Prep. Biochem. 4, 341-352.
Myles, D.G., Primakofi, P. \& Bellve, A.K. (1981) Surface domains of the guinea-pig sperm defined with monoclonal antibodies. Cell 23, 433-439.

Neville, D.M. (1976) The preparation of cell surface membrane enriched fractions. In Biochemical Analysis of Membranes, pp. 27-54. Ed. A. H. Maddy. J. Wiley \& Sons Inc., New York.

Noland, T.D., Olson, G.E. \& Garbers, D.L. (1983) Purification and partial characterization of plasma membranes from bovine spermatozoa. Biol. Reprod. 29, 987-998.

Olson, G.E., Hamilton, D.W. \& Fawcett, D.W. (1976) Isolation and characterization of the fibrous sheath of rat epididymal spermatozoa. Biol. Reprod. 14, $517-530$.

Peterson, R., Russell, L., Bundman, D. \& Freund, M. (1980) Evaluation of the purity of boar sperm plasma membranes prepared by nitrogen cavitation. Biol. Reprod. 23, 637-645.

Towbin, H., Staehelin, T. \& Gordon, J. (1979) Electrophoretic transfer of proteins from polyacrylamide gels to nitrocellulose sheets: procedure and some applications. Proc. natn Acad. Sci. U.S.A. 76, $4350-4354$.

Zahler, W.L. \& Doak, G.A. (1975) Isolation of outer acrosomal membranes from bull sperm. Biochim. Biophys. Acta 406, 479-488. 